\title{
Phenotypes, genotypes, and prevalence of congenital myopathies older than 5 years in Denmark
}

\section{OPEN}

Nanna Witting, MD,

$\mathrm{PhD}$

Ulla Werlauff, PT, PhD

Morten Duno, PhD

John Vissing, MD,

DMSci

Correspondence to

Dr. Witting:

nanna.witting@regionh.dk
ABSTRACT

Objective: Congenital myopathy as a nosologic entity has long been recognized, but knowledge of overall and subtype prevalence and phenotype-genotype relationship is scarce, especially in the adult population.

Methods: A national cohort of 107 patients $\geq 5$ years diagnosed with congenital myopathy were prospectively assessed clinically, histologically, and genetically.

Results: Twenty-five patients were excluded because of atypical features or alternative etiologies. The remaining 82 were on average 28 years old. Histologic examination revealed 14 (17\%) with core disease, 15 (18\%) centronuclear myopathy, 12 (15\%) nemaline rods, 27 (33\%) congenital fiber-type disproportion or type I predominance, and 14 (17\%) nonspecific myopathic changes. Genetic etiology was identified in 46 patients (56.1\%); $22.0 \%$ were heterozygous or compound heterozygous for mutations in RYR1, 7.3\% had DNM2 mutations, and 7.3\% NEB mutations. Less than 5\% had mutations in ACTA1, TPM2/3, MTM1, TTN, SEPN1, or SC4NA. A genetic cause was established in $83 \%$ with specific histology (cores/rods/centronuclear myopathy) vs 29\% with unspecific histology. The detailed clinical examination found gene-dependent discrepancies in the pattern of muscle affection and walking ability. Although walking ability was delayed in patients with ACTA1, TPM2/3, and RYR1 mutations, it was within normal limits in patients with NEB and DNM2 mutations.

Conclusions: We found that overall, genetic and histologic prevalence of congenital myopathy in Denmark differs from previous retrospective reports. Less RYR1 and more DNM2 and NEB mutations and less core histology were present in our cohort. These differences may be explained by our prospective design, the older cohort of patients, and by differences in genetic background. Neurol Genet 2017;3:e140; doi: 10.1212/NXG.0000000000000140

\section{GLOSSARY}

$\mathbf{C C D}=$ central core disease; $\mathbf{C F T D}=$ congenital fiber-type disproportion; $\mathbf{C M}=$ congenital myopathy; $\mathbf{C M S}=$ congenital myasthenic syndrome; CNM = centronuclear myopathy; DNM2 = dynamin 2; HGMD = Human Gene Mutation Database; LGMD = limb-girdle muscular dystrophy; MRC = Medical Research Council; MTM1 = myotubularin 1; NM = nemaline myopathy; RYR1 = ryanodine receptor 1 ; SEPN1 = selenoprotein 1; WES = whole-exome sequencing.

Congenital myopathy (CM) has been recognized for decades, but the genetic cause is only established in about half of cases ${ }^{1,2}$ and the knowledge of the distribution of genetic and histologic subtypes is insufficient. Data concerning CM have been collected retrospectively and mainly in pediatric patients.

The low genetic identification rate can partly be explained by undefined genes and by that some of the genes associated with $\mathrm{CM}^{3-6}$ are very large, making analyses challenging. An uncertain relationship between the genotype and the clinical/histologic phenotype also hampers the identification of genetic etiology. CM is traditionally classified according to muscle histology into nemaline, core, or centronuclear myopathies (CNMs). It has become increasingly clear,

See editorial

From the Copenhagen Neuromuscular Center (N.W., J.V.), Department of Neurology, and Department of Clinical Genetics (U.W.), Rigshospitalet, University of Copenhagen; and The Danish National Rehabilitation Centre for Neuromuscular Diseases (M.D.), Aarhus, Denmark. Funding information and disclosures are provided at the end of the article. Go to Neurology.org/ng for full disclosure forms. The Article Processing Charge was paid by the authors.

This is an open access article distributed under the terms of the Creative Commons Attribution-NonCommercial-NoDerivatives License 4.0 (CC BY-NC-ND), which permits downloading and sharing the work provided it is properly cited. The work cannot be changed in any way or used commercially without permission from the journal. 
however, that not only can CMs be associated with unspecific pathologies but the pathology and genotype are not mutually specific. ${ }^{7} \mathrm{~A}$ better understanding of prevalence and relationship between phenotypes and genotypes is a prerequisite for patient care, corroboration genetic findings, and ultimately development of treatment. In Denmark, patients with CM are registered at 2 centers. We made use of this opportunity to prospectively evaluate phenotypes and genotypes in a national cohort with a diagnosis of CM.

METHODS The study was conducted at the Copenhagen Neuromuscular Center, Rigshospitalet, Denmark, in collaboration with the Danish National Rehabilitation Centre for Neuromuscular Diseases. One hundred nineteen patients registered with a diagnosis of $\mathrm{CM}$ aged older than 5 years were invited. Two patients were not invited because of severe psychiatric comorbidity. The age limit of 5 years was chosen, as the functional tests used were not validated for younger patients and because we wanted to focus on older CMs. All participants completed questionnaires concerning symptoms and medical history. A neurologist (N.W.) and a physiotherapist (U.W.) examined all patients. Muscle strength was evaluated using a transformed 11-point Medical Research Council (MRC) scale $(0-10) .{ }^{8}$ Creatine kinase was assessed and DNA was isolated. After initial evaluation, participants with phenotypic characteristics atypical for $\mathrm{CM}$ (adult onset, fast progression, creatine kinase $\geq 600 \mathrm{U} / \mathrm{L}$, dystrophy as the main histologic finding, or alternative disease explanation) were excluded. Participants with a CM phenotype, but other genetic etiology, were excluded in the course of the genetic evaluation.

Standard protocol approvals, registrations, and patient consents. The study was approved by the local ethics committee (protocol H-C-2009-017), and all participants or their parents provided informed consent.

Genetic test strategy. All participants were tested sequentially for mutations in skeletal muscle alpha actin 1 (ACTA1) and tropomyosin $2+3(T P M 2+3)$ genes, as no definite histologic or clinical phenotypes are established for these genes, and the tests were readily available. Second-line analyses were directed by specific histologic or phenotypic findings. Core histology (central core disease [CCD]) elicited testing of the ryanodine receptor $1(R Y R 1)$ gene and the selenoprotein 1 (SEPN1) gene. CNM or pronounced ophthalmoplegia leads to assessment of the genes dynamin 2 (DNM2), myotubularin 1 (MTM1), amphiphysin (BIN1), and RYR1. Rigid spine patients went through SEPN1 testing. Pronounced contractures led to the investigation of collagen VI genes (not considered $\mathrm{CM}$ by the authors). The remaining unclassified patients were examined for aberrations in $N E B$ and $R Y R 1$, and if no mutations were found, exome sequencing was performed in the majority (Broad Institute, Boston or Nijmegen University, Holland) with subsequent assessment of genes involved in myopathy.

Genetic analyses. DNA was isolated from blood. The exons and flanking sequences of ACTA (NM_001100.3), TPM2 (NM_003289.3), and TPM3 (NM_152263.2) were PCR amplified and Sanger sequenced using BigDye v1.1 on an ABI3130 sequencer. Analysis of DNM2 is described elsewhere. ${ }^{7}$ The NEB (NM_004543.4) and RYR1 (NM_000540.2) genes were sequenced using a custom AmpliSeq targeting approach on an Ion PGM (Thermo Fisher). Areas with low $(<30 \mathrm{X})$ or missing coverage were Sanger sequenced. Data analysis was performed on the Torrent Suite v.3.6 or higher. All variants were confirmed by Sanger sequencing.

RESULTS Participants. One hundred seven of the 119 invited participants were included (figure 1). Twenty-five were excluded, leaving a total of 82 in the study. Of the excluded, 14 displayed a phenotype inconsistent with CM; 3 of these were subsequently confirmed with limb-girdle muscular dystrophy, type 1C (LGMD1C), LGMD2L, and LGMD2A. Eleven had a phenotype compatible with CM, but an alternative genetic etiology was identified in 10 , and DNA was missing in 1 (figure 1).

Prevalence and distribution of histology. Denmark has 5.4 million inhabitants older than 5 years. With 82 CM cases, prevalence is estimated to $2: 100,000$ in persons older than 5 years.

Forty-one had specific histology; 14 (17\%) had cores (3 multimini core disease), 15 (18\%) had CNM, and 12 (15\%) nemaline myopathy (NM). The remaining 41 had more unspecific histology; 27 (33\%) had congenital fiber-type disproportion (CFTD) or type I predominance (T1), and 14 (17\%) had unspecific myopathic biopsies. In 2, biopsy material was unavailable (table 1 ).

Genetics. A genetic diagnosis was reached in 46 (56.1\%) (tables 1 and 2). Eighteen had mutations in RYR1 (22.0\%) (13 heterozygous and 5 compound heterozygous), 6 had mutations in DNM2, and 6 in $N E B$. Three or less had mutations in ACTA1, TPM2, TPM 3, MTM1, SEPN1, SCN4A, or TTN genes (tables 1 and 3 ). Twenty-one participants had a particular clinical/histologic phenotype leading to a genetic diagnosis. Twenty-five patients had no clinical or histologic clues, and genetic etiology was identified by single gene testing in 20 and by wholeexome sequencing (WES) in 5; 3 with recessive RYR1 mutations, 1 with mutation in TTN, and 1 with mutation in SCN4A.

The diagnostic yield was highly dependent on histologic findings (table 2); if only participants with cores, CNM, or NM were evaluated, a genetic etiology was identified in $83 \%$. If CFTD/T1 were included, the number decreased to $63 \%$.

A genetic etiology was identified in 3/14 (21\%) with unspecific histology, 9/27 (33\%) with CFTD/ T1, 12/12 (100\%) with NM, 9/14 (64\%) with cores, and $13 / 15$ (87\%) of participants with CNM. CFTD and $\mathrm{T} 1$ were pooled, as these histologies coexisted in the same families. 


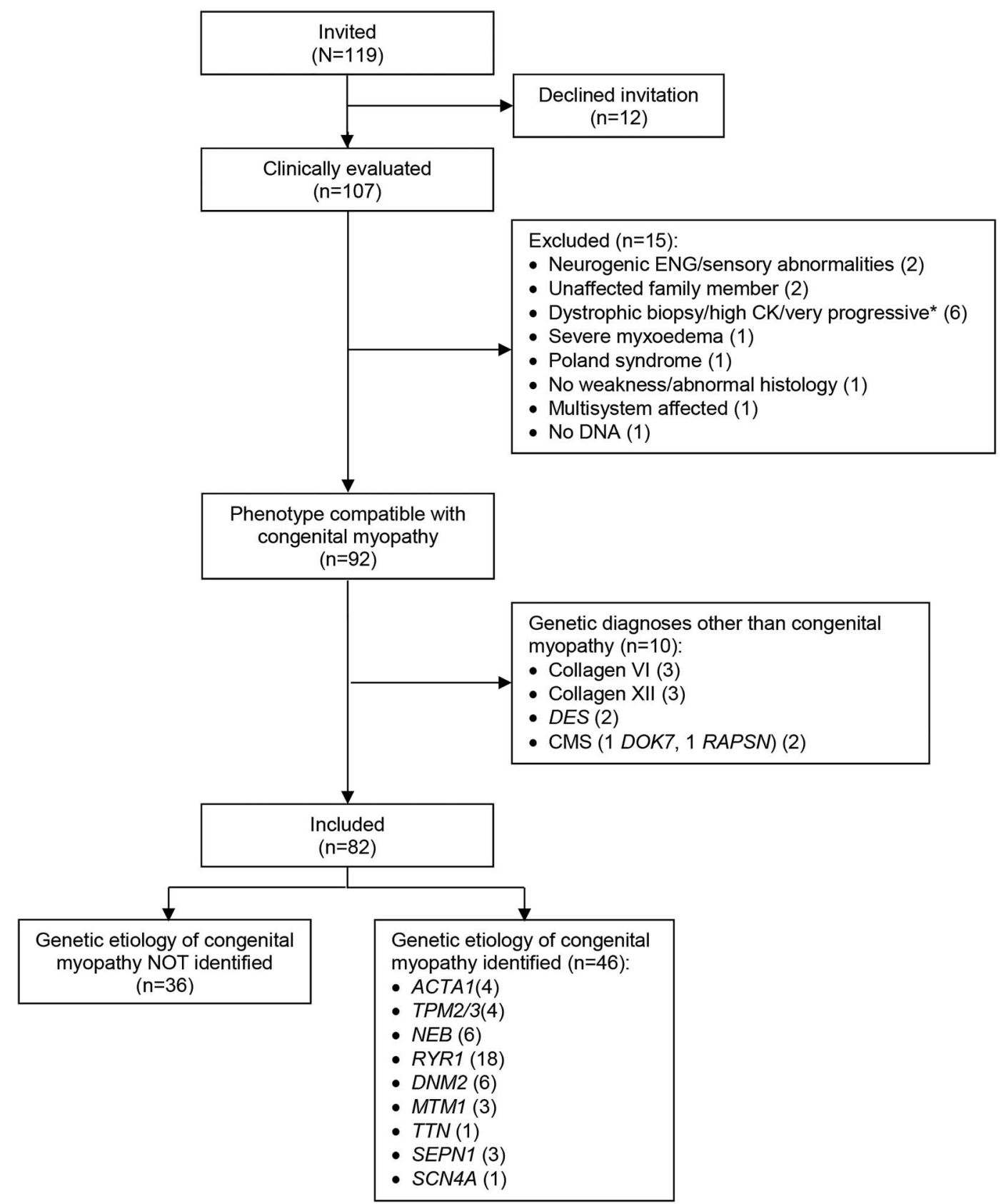

ACTA1 = skeletal muscle $\alpha$-actin $1 ; A D=$ autosomal dominant; $A R=$ autosomal recessive; $C K=$ creatine kinase; $C M S=$ congenital myasthenic syndrome; DES = desmin; DNM2 = dynamin 2; DOK7 = downstream-of-kinase 7 myasthenic syndrome; LGMD = limb-girdle muscular dystrophy; MTM1 = myotubularin 1; NEB = nebulin; RAPSN = receptor-associated protein of the synapse myasthenic syndrome; RYR1 = ryanodine receptor $1 ; \operatorname{SCN} 4 \mathrm{~A}=$ sodium channel $4 \mathrm{~A}$; SEPN1 = selenoprotein $1 ;$ TPM2/3 = tropomyosin 2/3; TTN = titin. *Of these 6, 1 had LGMD2L, 1 LGMD2A, and 1 LGMD1C.

The 10 participants with a phenotype compatible with CM, but alternative genetic etiology had collagen myopathy, desminopathy, or congenital myasthenic syndrome (CMS) (figure 1). Seven of 10 had unspecific or CFTD histology, whereas the remaining 3 had more specific histology: one with desminopathy had cores, another with desminopathy had rods, and one collagen VI had centronuclear changes (table 2).
The age of genetically unresolved $(27.8 \pm 16.7)$ and resolved $(28.0 \pm 14.6)$ patients was identical. The genetically unresolved had generally more unspecific histology and were less severely affected (tables 2 and 3).

Mutations. A total of 48 different mutations were identified of which 31 were listed in the Human Gene Mutation Database (HGMD) or ClinVar as pathogenic. The remaining 17 were absent from the 


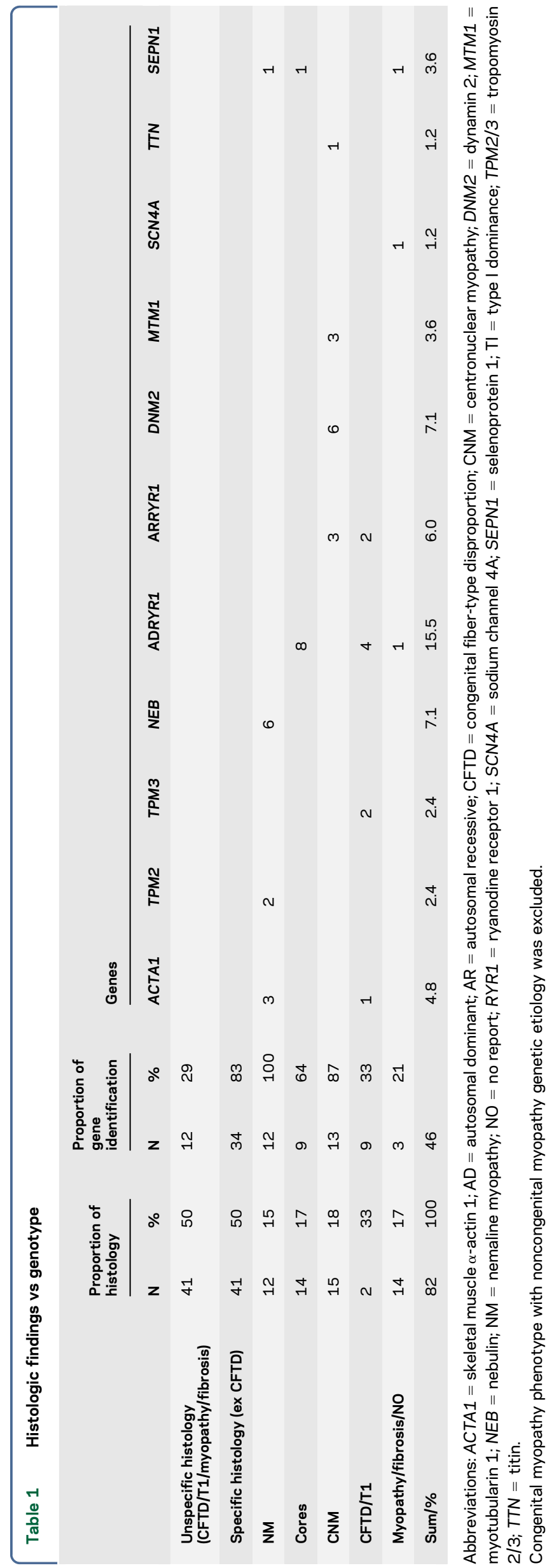

HGMD or ClinVar and to our knowledge have not been associated with disease before. Twelve of these were identified in recessively inherited disorder; 8 were predicted to result in premature stop codons or frameshifts and 4 were missense mutations. The 4 missense mutations were identified in SEPN1, RYR1, and SCN4A, respectively, where mutations very often are missense and were predicted to be potentially pathogenic by SIFT, PolyPhen2, AlignGVGD, and MutationTaster in silico prediction softwares and were absent from the ExAC database compiling information of more than 120,000 alleles (exac.broadinstitute.org), and from a Danish control cohort of 2000 WES. ${ }^{9}$ Of the 4 variants, 2 were identified along with a known pathogenic mutation, whereas the remaining 2 were identified in SEPN1 in a patient with a clear clinical presentation of a selenoprotein deficiency. Where family members were available, segregation analyses were performed to conform a compound heterozygous state.

The remaining 5 novel variants were all missense variants in RYR1 located in the well-known hotspot region for the dominantly inherited RYRI disorder. ${ }^{10}$

Phenotypes. From the detailed muscle examination, some patterns of weakness could be recognized for particular genotypes (table 3, figure 2). The $A C$ $T A 1$ patients were generally very weak in proximal, distal, and respiratory muscles, although 1 adult patient did not follow this pattern. By contrast, the TPM $/ 3$ patients had few MRC measurements below 7, but despite this had the same decrease in respiratory function as the $A C T A 1$ patients. NEB patients typically had preferential affection of the shoulder girdle and pronounced ankle dorsiflexor weakness with MRC of just 1-2, but comparatively mild respiratory involvement. As expected, dominant and recessive $R Y R 1$ patients differed in severity, with recessive patients being more severely affected. The DNM2 patients had a relatively nonselective profile except a marked ankle weakness. The MTM1 patients were more severely affected than the others, but hand function was relatively better than that in the ACTA1 patients. The respiratory involvement in the MTM1 and SEPN1 patients exceeded that in all other groups. Finally, scoliosis was noted in the SEPN1 patients, but otherwise only occasionally. Contractures were not prevalent, but were noted in some $N E B, R Y R 1$, DNM2, MTM1, TTN, and SEPN1 patients (table 3). Ophthalmoplegia was confined to DNM2 and MTM1 and recessive RYR1. Ptosis was mostly seen in DNM2 patients. Average time of walking ability was calculated in groups with more than 3 patients and exceeded the World Health Organizationdefined normal limit of 18 months in patients with 
Table 2 Chance of identifying genetic etiology according to histology

\begin{tabular}{|c|c|c|c|c|c|c|c|}
\hline & \multirow{2}{*}{\multicolumn{2}{|c|}{$\begin{array}{l}\text { Proportion of } \\
\text { histology }\end{array}$}} & \multicolumn{5}{|c|}{ Proportion of gene identification } \\
\hline & & & \multicolumn{2}{|c|}{ CM gene } & \multicolumn{2}{|c|}{$\begin{array}{l}\text { Non-CM } \\
\text { gene }\end{array}$} & \multirow[b]{2}{*}{ Genes } \\
\hline & $\mathbf{N}$ & $\%$ & $N$ & $\%$ & $\mathbf{N}$ & $\%$ & \\
\hline $\begin{array}{l}\text { Unspecific histology } \\
\text { (CFTD/T1/myopathy/fibrosis) }\end{array}$ & 49 & & 12 & 25 & 7 & 15 & \\
\hline Specific histology (ex CFTD) & 44 & & 34 & 77 & 3 & 7 & \\
\hline NM & 13 & 14 & 12 & 92 & 1 & 8 & DES \\
\hline Cores & 15 & 16 & 9 & 60 & 1 & 7 & DES \\
\hline CNM & 16 & 17 & 13 & 81 & 1 & 6 & ColVI \\
\hline CFTD/T1 & 32 & 35 & 9 & 28 & 5 & 16 & 2 CoIVI, 3 ColXII \\
\hline Myopathy/fibrosis/NO & 16 & 17 & 3 & 19 & 2 & 13 & 1 DOK7, 1 RAPS \\
\hline Sum/\% & 92 & 100 & & & & & \\
\hline
\end{tabular}

Abbreviations: CFTD = congenital fiber-type disproportion; $\mathrm{CM}=$ congenital myopathy; $\mathrm{CNM}=$ centronuclear myopathy; $\mathrm{NM}=$ nemaline myopathy; $\mathrm{NO}=$ no report; $\mathrm{TI}=$ type I dominance.

$\mathrm{CM}$ phenotype with noncongenital genetic etiology was included.

mutations in ACTA1 (30 months), TPM2/3 (20 months), and RYR1 (21 months, 2 never walked), whereas patients with mutations in $N E B$ (15 months) and DNM2 (14 months) fell within normal development. Four patients (a genetically unresolved participant aged 26 years, 1 DNM2 patient aged 69, an $R Y R 1$ patient aged 55 years, and a TTN patients aged 13 years) died during the study period from 2009 until now.

Information on work history was available in 42 of the 46 genetically verified cases; 36 attended school or worked part or full time. The remaining 6 received pension. No participant had clinical evidence of cardiac involvement, and ECG performed in nearly all participants was unremarkable.

DISCUSSION The present study investigated patients with CM older than 5 years. As only survivors from the early childhood were included, the information gathered is helpful for health care personal caring for older children and adults because existing knowledge has been obtained preferentially from pediatric cohorts. The study is also a prospective, national study of phenotypes and genotypes in CM. Therefore, unlike previous retrospective studies, the study is less affected by selection bias and strengthened by a systematic data collection by just 1 neurologist and physiotherapist. The report presents new data on national prevalence, distribution of histologic subtypes and genotypes, and expands the description of phenotypic characteristics. Because of the relatively low number of participants, the data need confirmation.

We estimated a prevalence of 2:100,000. This is lower than most previous studies, which determined the prevalence by chart reviews or databases to about 4:100,000 and focused on the pediatric population. ${ }^{11-13}$ The exclusion of children not surviving to 5 years in our study could account for some of the discrepancy. A previous study reported that $12 \%$ of their patients with $\mathrm{CM}$ died before the age of 6 years. Those patients had mutations in ACTA1, MTM1, or KLHL40.2 Patients with mutations in those genes may therefore be underrepresented in our material if extrapolated to a general population. The total prevalence, however, is probably not much influenced by this, as we, compared to previous studies, have included more elderly persons and in that way "compensate" for the lack of young children. Another explanation for our lower prevalence estimate could be that the prospective evaluation in our study may have eliminated more wrongly diagnosed patients. This is, however, probably not the main explanation for the discrepancy, as most of the studies were very thorough in the attempt to avoid misdiagnosis. ${ }^{11-13}$ Alternatively, our very stringent inclusion criteria may have omitted a few CMs with atypical features like high creatine kinase. Of the 15 excluded cases because of atypical features, however, 10 had definitely not $\mathrm{CM}$, as alternative etiologies were identified or they turned out to be asymptomatic family members.

Citizens in Denmark are easily traceable, as they are centrally registered, and therefore, the lower prevalence in our study is not likely caused by problems in identifying patients. A number of inherited muscle diseases, such as some limb-girdle muscular dystrophies $^{14}$ and myotonic dystrophy type 2 , differ markedly in prevalence in Denmark vs other countries, and this could also be the case for CMs. In support of this, $\mathrm{CM}$ has the same low prevalence in Northern England (1.37:100,000, including Bethlem myopathy 
Table 3 Characteristics of patients with or without genetic diagnosis

\begin{tabular}{|c|c|c|c|c|c|c|c|c|c|c|c|}
\hline ID/sex/age at examination, $y$ & Gene/mutation & $\begin{array}{l}\text { Protein } \\
\text { consequence }\end{array}$ & Histology & Course & $\begin{array}{l}\text { Walk, } \\
\text { mo old }\end{array}$ & $\begin{array}{l}\text { Ophthalmoplegia/ } \\
\text { ptoses }\end{array}$ & $\begin{array}{l}\text { Face/voice/palate/facial } \\
\text { palsy/neck }\end{array}$ & Limbs & $\begin{array}{l}\text { FVC } \% \\
\text { exp }\end{array}$ & $\begin{array}{l}\text { Contractures/ } \\
\text { scoliosis/other }\end{array}$ & Occupation \\
\hline \multicolumn{12}{|l|}{$\begin{array}{l}\text { Genetically diagnosed } \\
\text { patients }\end{array}$} \\
\hline ACTA1 & NM_001100.3 & & & & & & & & & & \\
\hline $16 / \mathrm{M} / 23$ & c. $16 \mathrm{G}>\mathrm{A}$ & p.Glu6Lys & CFTD & $\rightarrow$ & 24 & $\mathrm{~N} / \mathrm{N}$ & Oblong/NV/H/F/StW, NK6 & $\begin{array}{l}\text { UL 7P, 9D, LL } \\
7 P=D\end{array}$ & 47 & & $\mathrm{MD}$ \\
\hline 42/M/12 & c. $128 \mathrm{~A}>\mathrm{G}$ & p.Gln43Arg & $\mathrm{TI}$ & $\rightarrow$ & 42 & $N / N$ & $\begin{array}{l}\text { Dysarthria, short frenulum/ } \\
\mathrm{H} / \mathrm{F}+++/ \mathrm{NK} 4\end{array}$ & $\begin{array}{l}\text { UL 3P, 7D, LL 2P, } \\
6 \mathrm{D}\end{array}$ & 90 & & Student \\
\hline 44/M/15 & c. $142 \mathrm{G}>\mathrm{A}$ & p.Gly48Ser & CFTD & $\uparrow$ & 30 & $N / N$ & H/F/NK6 & $\begin{array}{l}\text { UL 5P, 9D, LL 6P, } \\
9 \mathrm{D}\end{array}$ & 55 & & Student \\
\hline 49/F/10 & c. $1106 \mathrm{C}>\mathrm{T}$ & p.Pro369Leu & TI/NM & $\rightarrow$ & 24 & $\mathrm{~N} / \mathrm{N}$ & $\begin{array}{l}\text { H/NK6/dysphagia, tired } \\
\text { chewing }\end{array}$ & $\begin{array}{l}\text { UL 4P, 6D, LL 5P, } \\
9 \mathrm{D}\end{array}$ & 53 & & School \\
\hline TPM2 & NM_003289.3 & p.Glu6Lys & & & & & & & & & \\
\hline 34/F/14 & c.415_417delGAG & p.Glu139del & NA & $\rightarrow$ & 23 & $N / Y$ & $\begin{array}{l}\text { Oblong/H/F/NK } 7 / \text { tired } \\
\text { chewing }\end{array}$ & $\begin{array}{l}\text { UL 5P, 9D, LL 5P, } \\
8 D\end{array}$ & 58 & $\mathrm{~s}$ & Student \\
\hline 35/F/39 & 34 daughter & p.Glu139del & $\begin{array}{l}\text { NM/ } \\
\text { CFTD }\end{array}$ & $\rightarrow$ & 15 & $N / Y(S u)$ & $\begin{array}{l}\text { Oblong/H/FNK6/tired } \\
\text { chewing }\end{array}$ & $\begin{array}{l}\text { UL 6P, 9D, LL } \\
8 \mathrm{P}=\mathrm{D}\end{array}$ & 74 & Pes cavus & $\mathrm{NI}$ \\
\hline ТРМЗ & NM_152263.2 & & & & & & & & & & \\
\hline $5 / M / 27$ & c. $503 \mathrm{G}>\mathrm{A}$ & p.Arg168His & CFTD & $\rightarrow$ & 17 & $N / N$ & Triangular/NV/H++/NK7 & $\begin{array}{l}\text { UL 8P, 10D, LL } \\
10 P, 9 D\end{array}$ & 46 & (C)/(S) & IT expert \\
\hline 91/M/19 & c. $502 \mathrm{C}>\mathrm{T}$ & p.Arg168Cys & CFTD & $\uparrow$ & 24 & $\mathrm{~N} / \mathrm{N}$ & $\begin{array}{l}\text { Oblong/NV, dysarthria/H/ } \\
\text { NK6, malocclusion }\end{array}$ & $\begin{array}{l}\text { UL 7P, 9D, LL 8P, } \\
\text { 7D }\end{array}$ & 57BiPAP & $\mathrm{RN}$ & Student \\
\hline NEB & NM_004543.4 & & & & & & & & & & \\
\hline $7 / F / 29$ & $\begin{array}{l}\text { c. } 2836-2 A>G^{a} \\
\text { c. } 5763+5 G>A\end{array}$ & p.?; p.? & NM & $\downarrow$ & 22 & $\mathrm{~N} / \mathrm{N}$ & H/F/NK7/dysphagia & $\begin{array}{l}\text { UL 5P, 8D, LL 4P, } \\
6 \mathrm{D}\end{array}$ & 77 & $\begin{array}{l}\text { /S/Rec patella lux } \\
\text { (Su) }\end{array}$ & Part-time job \\
\hline $18 / \mathrm{M} / 27$ & Brother to 53 & & NM & $\rightarrow$ & 15 & $N / N$ & Oblong/F/NK10 & $\begin{array}{l}\text { UL 7P, 9D, LL 8P, } \\
\text { 9D }\end{array}$ & 72 & & Economist \\
\hline $53 / \mathrm{M} / 24$ & $\begin{array}{l}\text { c. } 2415+1 G>A \\
\text { c. } 2415+1 G>A\end{array}$ & p.?; p.? & ND & $\rightarrow$ & 12 & $N / N$ & H/F/NK10 & $\begin{array}{l}\text { UL 6P, 8D, LL } \\
9 P=D\end{array}$ & 83 & Hyperlax FE & Shop ass \\
\hline $47 / F / 26$ & $\begin{array}{l}\text { c. } 10354 \mathrm{~T}>\mathrm{C}_{i} \\
\text { c. } 17725 \mathrm{G}>\mathrm{T}\end{array}$ & $\begin{array}{l}\text { p.Tyr3452His; p. } \\
\text { Glu5909* }\end{array}$ & NA & $\downarrow$ & 14 & $N / N$ & $\begin{array}{l}\text { Retrognathia(Su)/NV/H/F/l } \\
\text { NK5/dysphagia }\end{array}$ & $\begin{array}{l}\text { UL 7P, 10D, LL } \\
\text { 7P, 3D }\end{array}$ & 83 & $\mathrm{~A}(\mathrm{Su}) / / \operatorname{Rec} \mathrm{K} \operatorname{lux}(\mathrm{Su})$ & Pensioner \\
\hline $48 / F / 7$ & $\begin{array}{l}\text { c.11330dup } \\
\text { c.10354T }>C\end{array}$ & p.?; p.Tyr3452His & NM & $\rightarrow$ & 12 & $N / N$ & H/F/NK7 & $\begin{array}{l}\text { UL 6P, 6D, LL 7P, } \\
4 \mathrm{D}\end{array}$ & 86 & & School \\
\hline $58 / \mathrm{M} / 49$ & $\begin{array}{l}\text { c. } 12130 C>T_{;} \\
\text {c.17503_17505 } \mathrm{del}^{\mathrm{a}}\end{array}$ & $\begin{array}{l}\text { p.Arg4044*; p. } \\
\text { Asp5835del }\end{array}$ & NA & & Late & $N / N$ & H/NK9 & $\begin{array}{l}\text { UL 8P, 7D, LL } \\
10 P, 5 D\end{array}$ & 79 & $\mathrm{E}, \mathrm{FF} /$ & Full-time work \\
\hline RYR1AD & NM_000540.2 & & & & & & & & & & \\
\hline $6 / F / 36$ & c. $14818 \mathrm{G}>\mathrm{C}$ & p.Ala4940Thr & $\mathrm{CC} / \mathrm{NM}$ & $\rightarrow$ & 18 & $N / N$ & Oblong/NV/H/NK5 & $\begin{array}{l}\mathrm{UL} 8 \mathrm{P}=\mathrm{D}, \mathrm{LL} \\
7 \mathrm{P}, 8 \mathrm{D}\end{array}$ & 62 & $\mathrm{~A} / \mathrm{S}(\mathrm{Su})$ & Office \\
\hline $24 / M / 22$ & c. $14582 \mathrm{G}>\mathrm{A}$ & p.Arg4861His & $\mathrm{CC} ?$ & $\rightarrow$ & Never & $N / N$ & $\mathrm{H} /(\mathrm{F}) / \mathrm{NK} 5$ & $\begin{array}{l}\text { UL 5P, 7D, LL 2P, } \\
6 D\end{array}$ & 62 & $\mathrm{~K}, \mathrm{HF}(\mathrm{Su}) / \mathrm{S}(\mathrm{Su})$ & Pensioner \\
\hline
\end{tabular}




\begin{tabular}{|c|c|c|c|c|c|c|c|c|c|c|c|}
\hline Table $3 \quad$ Continued & & & & & & & & & & & \\
\hline ID/sex/age at examination, y & Gene/mutation & $\begin{array}{l}\text { Protein } \\
\text { consequence }\end{array}$ & Histology & Course & $\begin{array}{l}\text { Walk, } \\
\text { mo old }\end{array}$ & $\begin{array}{l}\text { Ophthalmoplegial } \\
\text { ptoses }\end{array}$ & $\begin{array}{l}\text { Face/voice/palate/facial } \\
\text { palsy/neck }\end{array}$ & Limbs & $\begin{array}{l}\mathrm{FVC} \% \\
\exp \end{array}$ & $\begin{array}{l}\text { Contractures/ } \\
\text { scoliosis/other }\end{array}$ & Occupation \\
\hline $25 / F / 38$ & c. $14567 C>G$ & p.Ala4856Gly & $\mathrm{CC}$ & $\rightarrow$ & 14 & $\mathrm{~N} / \mathrm{N}$ & $(\mathrm{H}) / \mathrm{NK} 9$ & $\begin{array}{l}\text { UL 7P, 10D, LL } \\
7 P, 9 D\end{array}$ & 90 & & $\begin{array}{l}\text { Part-time } \\
\text { draftsman }\end{array}$ \\
\hline $31 / F / 27$ & $\begin{array}{l}\text { c. } 14422- \\
14423 \text { delins } A^{a}\end{array}$ & p.Phe4808Asn & $\mathrm{CC}$ & $\rightarrow$ & Never & $\mathrm{N} / \mathrm{N}$ & $\mathrm{NV} / \mathrm{H} /(\mathrm{F}) / \mathrm{NK} 7$ & $\begin{array}{l}\text { UL 5P, 8D, LL 3P, } \\
8 D\end{array}$ & 90 & Hip lux & Pensioner \\
\hline 33/F/14 & c. $7523 \mathrm{G}>\mathrm{A}$ & p.Arg2508His & $\mathrm{T} 1$ & $\rightarrow$ & 28 & $N / Y$ & $\begin{array}{l}\text { Oblong//NV, dysarthria/H/ } \\
\text { F/NK8 tired chewing }\end{array}$ & $\begin{array}{l}\text { UL/P, 10D, LL 7P, } \\
\text { 10D }\end{array}$ & & A/S/hyperlax & School \\
\hline $61 / \mathrm{M} / 62$ & c. $13913 G>A$ & p.Gly4638Asp & $\mathrm{CC}$ & $\rightarrow$ & 24 & $(Y) / Y$ & $\mathrm{NV} / \mathrm{H} / \mathrm{F} / \mathrm{NK} 9$ & $\begin{array}{l}\text { UL } 6 P, 10 D, L L \\
7 P, 8 D\end{array}$ & 94 & & Medical doctor \\
\hline $64 / F / 27$ & Daughter of 61 & & $\mathrm{CC}$ & $\rightarrow$ & 24 & $\mathrm{~N} / \mathrm{N}$ & $\mathrm{NV} / \mathrm{H} / \mathrm{NK} 10$ & $\begin{array}{l}\text { UL 7P, 10D, LL } \\
8 \mathrm{P}, 9 \mathrm{D}\end{array}$ & NA & A/hyperlordotic & Sales assistant \\
\hline $83 / \mathrm{M} / 63$ & c. $14567 \mathrm{C}>\mathrm{T}^{\mathrm{a}}$ & p.Ala4856Val & & $\rightarrow$ & 15 & $\mathrm{~N} / \mathrm{N}$ & (F)/NK10 & $\begin{array}{l}\text { UL 8P, 10D, LL } \\
8 \mathrm{P}=\mathrm{D}\end{array}$ & 97 & & Chauffeur \\
\hline $84 / M / 19$ & Son of 83 & & NA & $\uparrow$ & 20 & $\mathrm{~N} / \mathrm{N}$ & H/F)/NK10 & Normal & 100 & & $\begin{array}{l}\text { Electrician } \\
\text { trainee }\end{array}$ \\
\hline $62 / M / 18$ & c. $14567 \mathrm{C}>\mathrm{T}^{\mathrm{a}}$ & p.Ala4856Val & & $\uparrow$ & 18 & $\mathrm{~N} / \mathrm{N}$ & $\mathrm{NV} / \mathrm{H} / \mathrm{F} / \mathrm{NK} 10$ & Normal & 75 & & Student \\
\hline 89/M/31 & c. $14929 G>A^{a}$ & p.Glu4977Lys & M & $\rightarrow$ & 12 & $\mathrm{~N} / \mathrm{N}$ & Oblong/H/F/NK10 & $\begin{array}{l}U L 9 P=D, L L \\
10 P=D\end{array}$ & 77 & Cong hip lux & $\begin{array}{l}\text { Gardener, sick } \\
\text { leave }\end{array}$ \\
\hline $94 / \mathrm{M} / 22$ & c. $13891 \mathrm{~T}>\mathrm{C}^{\mathrm{a}}$ & p.Tyr4631His & $\begin{array}{l}\mathrm{CC} / \mathrm{NM} / \\
\text { CFTD }\end{array}$ & $\rightarrow$ & 32 & $N / N$ & $\mathrm{NV} / \mathrm{H} / \mathrm{NK} 9$ & $\begin{array}{l}\text { UL 5P, 7D, LL 6P, } \\
8 D\end{array}$ & 82 & $\mathrm{~A} / \mathrm{S} /$ hyperlax $\mathrm{E}+\mathrm{Fi}$ & $\begin{array}{l}\text { Full-time office } \\
\text { job }\end{array}$ \\
\hline $105 / F / 28$ & c. $479 A>G$ & p.Gly159Glu & $\mathrm{CC}$ & $\rightarrow$ & 24 & $\mathrm{~N} / \mathrm{N}$ & Oblong/NV/H/(F)/StW/NK6 & $\begin{array}{l}\text { UL 8P, 9D, LL 6P, } \\
\text { 9D }\end{array}$ & NA & $\begin{array}{l}\mathrm{A}, \mathrm{Aa}(\mathrm{Su}) / / \mathrm{RS}, \\
\text { hyperlax } \mathrm{Fi}\end{array}$ & Psychologist \\
\hline RYR1AR & NM_000540.2 & & & & & & & & & & \\
\hline $2 / \mathrm{M} / 50$ & $\begin{array}{l}\text { c. } 2427 \_2446 \text { dup }^{a} ; \\
\text { c. } 325 C>T\end{array}$ & $\begin{array}{l}\text { p.Pro816Hisfs*75; } \\
\text { p.Arg109Trp }\end{array}$ & $\mathrm{CN} / \mathrm{D}$ & $\rightarrow$ & 36 & $\mathrm{Y} / \mathrm{N}$ & $\mathrm{NV} / \mathrm{H}+++/ \mathrm{F} / \mathrm{NK} 6$ & $\begin{array}{l}\text { UL 4P, 8D, LL 4P, } \\
10 D\end{array}$ & 68 & & Full-time work \\
\hline 9/M/10 & $\begin{array}{l}\text { c. } 325 \mathrm{C}>\mathrm{T}_{i} \\
\text { c. } 2989 \mathrm{C}>\mathrm{T}\end{array}$ & $\begin{array}{l}\text { p.Arg109Trp; p. } \\
\text { Arg997* }\end{array}$ & CFTD & $\rightarrow$ & 15 & $\mathrm{Y} / \mathrm{Y}$ & $\begin{array}{l}\text { Triangular//H/(F)/NK3, tired } \\
\text { chewing }\end{array}$ & $\begin{array}{l}\text { UL 4P, 7D, LL 3P, } \\
9 \mathrm{D}\end{array}$ & 45 & & School \\
\hline $70 / \mathrm{M} / 22$ & $\begin{array}{l}\text { c. } 718 C>T_{i} \\
\text { c. } 2897 C>T^{a}\end{array}$ & $\begin{array}{l}\text { p.GIn240*; p. } \\
\text { Pro966Leu }\end{array}$ & CFTD & $\uparrow$ & 16 & $\mathrm{Y} / \mathrm{Y}$ & $\begin{array}{l}\mathrm{H} / \mathrm{F} / \text { tired chewing, } \\
\text { dysphagia, NK9 }\end{array}$ & $\begin{array}{l}\text { UL 6P, 10D, LL } \\
5 P, 9 D\end{array}$ & 74 & $\begin{array}{l}\text { A, HF//rec hip }+ \text { Sh } \\
\text { lux }\end{array}$ & $\mathrm{NI}$ \\
\hline $81 / F / 28$ & Sister to 107 & & ND & $\rightarrow$ & 15 & $\mathrm{~N} / \mathrm{N}$ & F/NK3 & $\begin{array}{l}\text { UL 3P, 9D, LL 2P, } \\
8 D\end{array}$ & 72 & & Social worker \\
\hline $107 / F / 33$ & $\begin{array}{l}\text { c. } 325 \mathrm{C}>\mathrm{T} ; \\
\text { c. } 7308 \_7309 \text { delTG }^{\mathrm{a}}\end{array}$ & p.Arg109Trp; p.? & $\mathrm{CN}$ & $\rightarrow$ & 24 & $\mathrm{Y} / \mathrm{N}$ & $\begin{array}{l}\mathrm{NV/H} / \mathrm{F} / \mathrm{NK} 2 / \text { tired chewing, } \\
\text { dysphagia }\end{array}$ & $\begin{array}{l}\text { UL 2P, 6D, LL 2P, } \\
8 \mathrm{D}\end{array}$ & NA & Wheelchair & $\begin{array}{l}\text { Part-time social } \\
\text { worker }\end{array}$ \\
\hline DNM2 & NM_004945.3 & p.? & & & & & & & & & \\
\hline $8 / M / 25$ & c. $1393 \mathrm{C}>\mathrm{T}$ & p.Arg465Trp & $\mathrm{CN}$ & $\rightarrow$ & 18 & $(Y) / Y$ & Oblong/F/NK7 & $\begin{array}{l}\text { UL 8P, 10D, LL } \\
10 P, 9 D\end{array}$ & 74 & & $\begin{array}{l}\text { Part-time } \\
\text { student }\end{array}$ \\
\hline $46 / \mathrm{M} / 19$ & c. $1840 \mathrm{G}>\mathrm{A}$ & p.Ala614Thr & $\mathrm{CN}$ & $\downarrow$ & 17 & $\mathrm{Y} / \mathrm{Y}$ & $\begin{array}{l}\text { Skewed cranium/NV, } \\
\text { dysarthria/H/F/NK3 }\end{array}$ & $\begin{array}{l}\text { UL 2P, 5D, LL 2P, } \\
5 \mathrm{D}\end{array}$ & 19 & $\mathrm{RN}$ & Pensioner \\
\hline $50 / F / 69$ & c. $1393 \mathrm{C}>\mathrm{T}$ & p.Arg465Trp & $\mathrm{CN}$ & $\rightarrow$ & & $(Y) / Y$ & F/NK6 & $\begin{array}{l}\text { UL 5P, 6D, LL 5P, } \\
\text { 1D }\end{array}$ & 81 & & Retired (died) \\
\hline
\end{tabular}




\begin{tabular}{|c|c|c|c|c|c|c|c|c|c|c|c|}
\hline ID/sex/age at examination, $y$ & Gene/mutation & $\begin{array}{l}\text { Protein } \\
\text { consequence }\end{array}$ & Histology & Course & $\begin{array}{l}\text { Walk, } \\
\text { mo old }\end{array}$ & $\begin{array}{l}\text { Ophthalmoplegia/ } \\
\text { ptoses }\end{array}$ & $\begin{array}{l}\text { Face/voice/palate/facial } \\
\text { palsy/neck }\end{array}$ & Limbs & $\begin{array}{l}\text { FVC \% } \\
\text { exp }\end{array}$ & $\begin{array}{l}\text { Contractures/ } \\
\text { scoliosis/other }\end{array}$ & Occupation \\
\hline $82 / F / 41$ & c. $1393 \mathrm{C}>\mathrm{T}$ & p.Arg465Trp & $\mathrm{CN}$ & $\rightarrow$ & 9 & $\mathrm{~N} / \mathrm{N}$ & $\mathrm{H} / \mathrm{F} / \mathrm{NK} 8$ & $\begin{array}{l}\text { UL 7P, 8D, LL 7P, } \\
4 D\end{array}$ & 68 & & $\begin{array}{l}\text { Part-time } \\
\text { teacher }\end{array}$ \\
\hline $67 / F / 20$ & c. $1102 \mathrm{G}>\mathrm{A}$ & p.Glu368Lys & $\mathrm{CN}$ & $\rightarrow$ & 14 & $(Y) /(Y)$ & Oblong//H/(F)/N/NK6 & $\begin{array}{l}\text { UL 6P, 7D, LL 7P, } \\
\text { 5D }\end{array}$ & 56 & A(Su), FF//RN & $\begin{array}{l}\text { Part-time } \\
\text { student }\end{array}$ \\
\hline $85 / F / 52$ & c. $1553 \mathrm{G}>\mathrm{A}$ & p.Arg518His & CN/CFTD & $\rightarrow$ & 12 & $\mathrm{Y} / \mathrm{N}$ & Oblong/NV/H/NK5 & $\begin{array}{l}\text { UL 9P, 8D, LL 8P, } \\
\text { 5D }\end{array}$ & 88 & & $\begin{array}{l}\text { Health care } \\
\text { worker }\end{array}$ \\
\hline MTM1 & NM_000252.2 & & & & & & & & & & \\
\hline $37 / M / 14$ & c. $1353+1 G>A$ & p.? & $\mathrm{CN}$ & $\rightarrow$ & $\mathrm{N}$ & $Y / Y$ & Oblong/NV/H/F/NK2 & $\begin{array}{l}\text { UL 2P, 5D, LL 2P, } \\
3 \mathrm{D}\end{array}$ & IV & IS(Su) & $\begin{array}{l}\text { Special terms } \\
\text { stud }\end{array}$ \\
\hline $80 / M / 18$ & c. $674 \mathrm{~T}>\mathrm{C}$ & p.lle225Thr & $\mathrm{CN}$ & $\rightarrow$ & 36 & $\mathrm{Y} / \mathrm{N}$ & F/NK2 & $\begin{array}{l}\text { UL 2P, 6D, LL 1P, } \\
3 \mathrm{D}\end{array}$ & 19 & A(Su)//wheelchair & $\begin{array}{l}\text { Special terms } \\
\text { stud }\end{array}$ \\
\hline $102 / M / 24$ & c. $1037 \mathrm{G}>\mathrm{C}$ & p.Trp346Ser & CN/CFTD & $\uparrow$ & 18 & $\mathrm{Y} / \mathrm{N}$ & Oblong/NV/H/(F)/NK4 & $\begin{array}{l}\text { UL 4P, 9D, LL 4P, } \\
8 D\end{array}$ & 25 & Pes cavus/clawfoot & Student \\
\hline SEPN1 & NM_020451.2 & $\mathrm{Er}$ & & & & & & & & & \\
\hline $17 / F / 34$ & $\begin{array}{l}\text { c. } 802 C>T_{i} \\
\text { c. } 1574 \mathrm{~T}>\mathrm{G}\end{array}$ & $\begin{array}{l}\text { p.Arg268Cys; p. } \\
\text { Met525Arg }\end{array}$ & NM/D & $\rightarrow$ & 12 & $N / N$ & NV/H/F/StW, NKO & $\begin{array}{l}\text { UL 7P, 8D, LL } \\
P=D=8\end{array}$ & IV & (C)/S(Su) & Part-time job \\
\hline $101 / F / 20$ & $\begin{array}{l}\text { c.893T }>C ; \\
\text { c.1396C }>T^{a}\end{array}$ & $\begin{array}{l}\text { p.Leu298Pro; p. } \\
\text { Arg466Trp }\end{array}$ & NA & $\downarrow$ & & $N /(Y)$ & $\begin{array}{l}\text { Triangular/NV/H/(F)/StW/ } \\
\text { NK2 }\end{array}$ & $\begin{array}{l}\text { UL 7P, 10D, LL } \\
4 \mathrm{P}, 9 \mathrm{D}\end{array}$ & 22 & IS(Su) & Pensioner \\
\hline $103 / F / 27$ & $\begin{array}{l}\text { c. } * 1107 \mathrm{~T}>\mathrm{C} \\
\text { c. } 943 \mathrm{G}>\mathrm{A}\end{array}$ & p.?; p.Gly315Ser & MCD & $\downarrow$ & 12 & $N / N$ & $\mathrm{NV} / \mathrm{H} /(\mathrm{F}) / \mathrm{StW}, \mathrm{NK} 4$ & $\begin{array}{l}\text { UL 6P, 1OP, LL } \\
4 \mathrm{P}, 9 \mathrm{D}\end{array}$ & 30BiPAP & $A, R N / S$ & Pensioner \\
\hline TTN & NM_133378.4 & $\mathrm{p} . ? ; \mathrm{p}$ & & & & & & & & & \\
\hline 14/M/13 & $\begin{array}{l}\text { c. } 16745 \mathrm{C}>\mathrm{G} \\
\text { c. } 97719 \mathrm{C}>\mathrm{G}^{\mathrm{a}}\end{array}$ & $\begin{array}{l}\text { p.Ser5582*; p. } \\
\text { Tyr32573* }\end{array}$ & $\mathrm{CN}$ & $\downarrow$ & Never & $N / N$ & $\begin{array}{l}\text { Retrognathia/NV/H++/(F)/ } \\
\text { NK3 }\end{array}$ & $\begin{array}{l}\text { UL 3P, 6D, LL 2P, } \\
4 \mathrm{D}\end{array}$ & NIV & Hand K(Su)/S(Su) & Dead \\
\hline SCN4A & NM_000334.4 & $\mathrm{P}$ & & & & & & & & & \\
\hline $52 / F / 30$ & $\begin{array}{l}\text { c. } 673 \mathrm{C}>\mathrm{T} ; \\
\text { c. } 3626 \mathrm{G}>\mathrm{T}^{\mathrm{a}}\end{array}$ & $\begin{array}{l}\text { p.Arg225Trp; p. } \\
\text { Cys1209Phe }\end{array}$ & M & $\rightarrow$ & 18 & $\mathrm{Y} / \mathrm{N}$ & Oblong/NV/H/F/StW, NK5 & $\begin{array}{l}\text { UL 7P, 10D, LL } \\
6 \mathrm{P}, \mathrm{OD}\end{array}$ & 82 & Hyperlordosis & $\begin{array}{l}\text { Laboratory } \\
\text { tech part time }\end{array}$ \\
\hline \multicolumn{12}{|l|}{$\begin{array}{l}\text { Characteristics of not } \\
\text { genetically diagnosed } \\
\text { patients }\end{array}$} \\
\hline 1/M/66 & NA & NA & $\begin{array}{l}\mathrm{CC}, \mathrm{NM} \\
\mathrm{Dy}\end{array}$ & $\downarrow$ & NA & $\mathrm{N}$ & $\mathrm{NV} / \mathrm{H}+++/ \mathrm{StW}, \mathrm{NK3}$ & $\begin{array}{l}\text { UL 6P, 9D, LL 5P, } \\
3 \mathrm{D}\end{array}$ & 70 & $\begin{array}{l}\text { Oromandibular, } \mathrm{FE}, \\
\text { A//pes cavus }\end{array}$ & Pensioner \\
\hline $54 / \mathrm{M} / 69$ & NA & NA & $\mathrm{CC}$ & $\rightarrow$ & NA & $\mathrm{N}$ & (F)/NK10 & $\begin{array}{l}\mathrm{UL} 8 \mathrm{P}, 9 \mathrm{D}, \mathrm{LL} \\
7 \mathrm{P}=\mathrm{D}\end{array}$ & 64 & $\begin{array}{l}\text { Oromandibular, Sh, } \\
\text { K, A, wrists }\end{array}$ & Pensioner \\
\hline $3 / F / 18^{b}$ & NA & NA & M & $\rightarrow$ & 20 & $\mathrm{~N}$ & NV/NK10 & 10 & 75 & & Student \\
\hline $4 / \mathrm{M} / 13$ & NA & NA & $\mathrm{T} 1$ & $\rightarrow$ & 20 & $\mathrm{~N}$ & NV/NK9 & $\begin{array}{l}\text { UL 9P, 10D, LL } \\
9 P=D\end{array}$ & 75 & & School \\
\hline $10 / M / 35^{b}$ & NA & NA & MCD & $\downarrow$ & NA & $\mathrm{Y} / \mathrm{N}$ & Oblong/NV/F/NK2 & $\begin{array}{l}\text { UL 2P, 7D, LL 2P, } \\
\text { 3D }\end{array}$ & IV & $\begin{array}{l}\text { (All joints)/S(Su)/ } \\
\text { wheelchair }\end{array}$ & Pensioner \\
\hline $12 / F / 59^{b}$ & NA & NA & M & $\downarrow$ & NA & $\mathrm{N}$ & /NK8 & $\begin{array}{l}\mathrm{UL} 6 \mathrm{P}, 7 \mathrm{D}, \mathrm{LL} \\
4 \mathrm{P}=\mathrm{D}\end{array}$ & 100 & $\mathrm{~A}(\mathrm{Su})$, plantarflex/ & Secretary PT \\
\hline
\end{tabular}




\begin{tabular}{|c|c|c|c|c|c|c|c|c|c|c|c|}
\hline Continued & & & & & & & & & & & \\
\hline ID/sex/age at examination, y & Gene/mutation & $\begin{array}{l}\text { Protein } \\
\text { consequence }\end{array}$ & Histology & Course & $\begin{array}{l}\text { Walk, } \\
\text { mo old }\end{array}$ & $\begin{array}{l}\text { Ophthalmoplegia/ } \\
\text { ptoses }\end{array}$ & $\begin{array}{l}\text { Face/voice/palate/facial } \\
\text { palsy/neck }\end{array}$ & Limbs & $\begin{array}{l}\text { FVC \% } \\
\exp \end{array}$ & $\begin{array}{l}\text { Contractures/ } \\
\text { scoliosis/other }\end{array}$ & Occupation \\
\hline 95/F/32 & NA & NA & Normal & $\rightarrow$ & 10 & N & /NK10 & $\begin{array}{l}\text { UL 9P, 10D, LL } \\
9 P=D\end{array}$ & 100 & & $\mathrm{NI}$ \\
\hline $56 / \mathrm{M} / 32$ & NA & NA & M & $\rightarrow$ & NA & N & /NK9 & $\mathrm{UL}=\mathrm{LL} 9 \mathrm{P}, 10 \mathrm{P}$ & NA & A, Fi, K//hyperlax & Draftsman \\
\hline $15 / F / 18^{c}$ & NA & NA & CFTD & $\rightarrow$ & 16 & $N / Y$ & $\mathrm{NV} / \mathrm{H} / \mathrm{NK} 10$ & Normal & 100 & IS & School \\
\hline $19 / M / 22^{b}$ & NA & NA & MCD & $\rightarrow$ & Never & $\mathrm{N} / \mathrm{N}$ & Oblong/H/NK3 & $\begin{array}{l}\text { UL 2P, 6D, LL 2P, } \\
5 \mathrm{D}\end{array}$ & 35 & $\begin{array}{l}\text { K, E, Fi, hip(Su), A, } \\
\mathrm{Aa} / \mathrm{S}(\mathrm{Su})\end{array}$ & Student \\
\hline $20 / M / 14^{b}$ & NA & NA & $\mathrm{T} 1$ & $\rightarrow$ & 20 & $N / Y$ & $\begin{array}{l}\text { Oval, retrognathia/NV/H/ } \\
\text { NK10 }\end{array}$ & $\begin{array}{l}\text { UL } 9 \mathrm{P}, 10 \mathrm{P}, \mathrm{LL} \\
10 \mathrm{P}=\mathrm{D}\end{array}$ & 100 & & School \\
\hline $22 / F / 28^{b, c}$ & NA & NA & $\mathrm{T} 1$ & $\rightarrow$ & 16 & $(Y) / Y$ & H/F/NK9 & $\begin{array}{l}\text { UL 6P, 10D, LL } \\
6 \mathrm{P}, 9 \mathrm{D}\end{array}$ & 73 & & Dietician PT \\
\hline $28 / F / 55^{b}$ & NA & NA & NA & $\rightarrow$ & NA & N & $(\mathrm{H}) / \mathrm{NK} 8$ & $\mathrm{UL}=\mathrm{LL} 8 \mathrm{P}, 10$ & 100 & $/(\mathrm{S})$ & Physiotherapist \\
\hline $39 / \mathrm{M} / 40$ & NA & NA & CFTD & $\rightarrow$ & NA & N & StW, NK10 & $\begin{array}{l}\text { UL 8P, 10D, LL } \\
9 P, 10 D\end{array}$ & 84 & & $\mathrm{NI}$ \\
\hline $51 / F / 35$ & NA & NA & CFTD & $\rightarrow$ & NA & N & NK6 & $\begin{array}{l}\text { Asym, UL P4, 9D, } \\
\text { LL P7, D10 }\end{array}$ & 82 & $\begin{array}{l}\mathrm{KF}, \mathrm{HF}(\mathrm{Su}), \mathrm{RN} / \mathrm{S} / \\
\text { hyperlax }\end{array}$ & $\begin{array}{l}\text { Psychologist } \\
\text { PT }\end{array}$ \\
\hline 72/F/38 & NA & NA & NA & $\rightarrow$ & 14 & N & $(\mathrm{H}) / \mathrm{StW}, \mathrm{NK} 8$ & 10 & 92 & $\mathrm{FF} / / \mathrm{rec}$ patella lux & Teacher PT \\
\hline 71/F/11 & NA & NA & $M$ & $\rightarrow$ & $13, A M$ & N & (H)/StW, NK5/tired chewing & $\begin{array}{l}\text { UL 5P, 10D, LL } \\
7 P, 6 D\end{array}$ & 52 & $\begin{array}{l}\mathrm{KF}, \mathrm{HF}, \mathrm{EF}, \mathrm{A}(\mathrm{Su}), \\
\text { thumb/S }\end{array}$ & Special school \\
\hline 29/F/41 & NA & NA & M & $\downarrow$ & NA & N & Oblong/NV/H/StW, NK9 & $\begin{array}{l}\text { UL 9P, 10D, LL } \\
9 P, 5 D\end{array}$ & 90 & $A$ & $\begin{array}{l}\text { Graphic } \\
\text { designer }\end{array}$ \\
\hline $32 / F / 21^{b}$ & NA & NA & CCD & $\uparrow$ & NA & N & Oblong/NV/H/F/NK6 & $\begin{array}{l}\text { UL 5P, 8D, LL 4P, } \\
8 P\end{array}$ & 82 & & Student \\
\hline $41 / M / 21^{b}$ & NA & NA & M & $\downarrow$ & NA & $N / Y$ & H/NK10 & $\begin{array}{l}\mathrm{UL} 9 \mathrm{P}=\mathrm{D}, \mathrm{LL} \\
8 \mathrm{P}, 9 \mathrm{D}\end{array}$ & 94 & A & Student \\
\hline $55 / F / 17^{b}$ & NA & NA & CFTD & $\rightarrow$ & NA & $N / Y$ & $N V / H / F / N K 8$ & $\begin{array}{l}\text { UL 6P, 9D, LL 8P, } \\
8 D\end{array}$ & 53 & $\mathrm{FF}, \mathrm{HF}, \mathrm{KE}, \mathrm{RN}, \mathrm{A} / \mathrm{S}$ & Student \\
\hline $59 / \mathrm{M} / 44^{\mathrm{b}}$ & NA & NA & CNM & $\rightarrow$ & 24 & $\mathrm{~N}$ & $(\mathrm{H} / \mathrm{F}) / \mathrm{NK} 4$ & $\begin{array}{l}\text { UL 5P, 9D, LL 3P, } \\
5 \mathrm{D}\end{array}$ & 72 & A, hyperlax & Jeweler \\
\hline 63/F/14 & NA & NA & Normal & $\rightarrow$ & NA & $N / Y$ & $\mathrm{NV} / \mathrm{H} / \mathrm{F}$ & NA & 60 & & Special school \\
\hline $65 / M / 26^{b}$ & NA & NA & M & $\rightarrow$ & 24 & N & Oblong/NV/(H)/NK10 & $\begin{array}{l}\text { UL 9P, 10D, LL } \\
9 P, 10 D\end{array}$ & 89 & Sh & $\mathrm{NI}$ \\
\hline $66 / F / 11^{c}$ & NA & NA & M & $\rightarrow$ & 34 & $\mathrm{~N}$ & $(\mathrm{H}) / \mathrm{NK} 9$ & $\begin{array}{l}\text { UL 7P, 5D, LL 8P, } \\
6 D\end{array}$ & NA & //Rec lux; K, A, Sh & Special school \\
\hline $68 / \mathrm{M} / 8^{\mathrm{b}}$ & NA & NA & M & $\uparrow$ & 23 & N & F/NK9, tired chewing & $\begin{array}{l}U L \mathrm{LP}=\mathrm{D}, \mathrm{LL} 9 \mathrm{P} \\
=\mathrm{D}\end{array}$ & 78 & & School \\
\hline $74 / M / 13^{b}$ & NA & NA & CFTD & $\rightarrow$ & 17 & $Y / Y$ & $\mathrm{H} / \mathrm{F} / \mathrm{NK} 10$ & $\begin{array}{l}\text { UL 8P, 9D, LL OP, } \\
8 D\end{array}$ & 91 & /S/hyperlax & School \\
\hline $75 / F / 25^{b}$ & NA & NA & NA & $\uparrow$ & NA & N & NV/F/NK10 & $\begin{array}{l}\text { UL 8P, 10D, LL } \\
10 P=D\end{array}$ & 65 & & Childcare PT \\
\hline
\end{tabular}



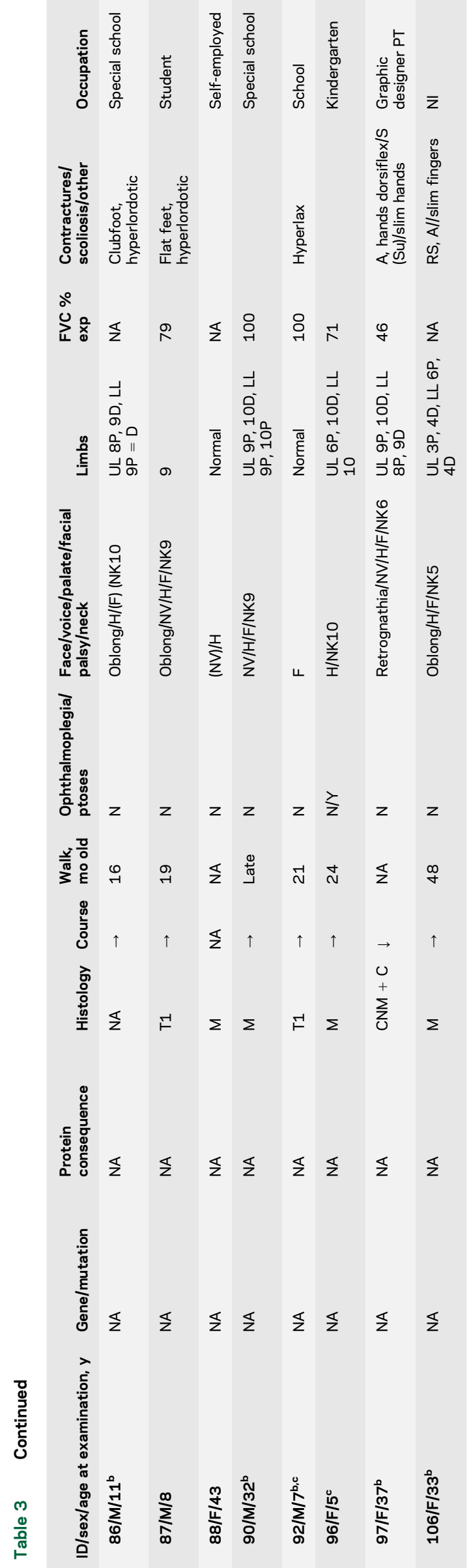

with a prevalence of 0.77 ) as in Denmark, where the genetic influence from historic Viking invasions is great. $^{15}$

A London-based study ${ }^{1}$ observed a different pattern of histology, as the majority of their patients had CCD, whereas histologic findings of cores, CNM, NM, and CFTD/T1 were more evenly distributed among our patients. This difference in histology also points to a different genetic background and supports that not only the prevalence but also the genetic make-up of CM varies among geographic regions. Concurring with this hypothesis, the genetic etiology in the London-based study was very different from ours. They found RYR1 mutations in $59 \%$ and DNM2 in $0 \%$ of their CMs, whereas RYR1 mutations were observed in $22 \%$ of our cases and DNM2 mutation in $7.3 \%$. The difference in $R Y R 1$ mutations in our population is probably not explained by different age distribution, as $R Y R 1$ patients usually survive past early childhood. ${ }^{2}$ The DNM2 patients, however, are relatively mildly affected and may go unrecognized for years, which would make them underrepresented in a very young population. We also found a higher percentage of patients with $N E B$ mutations in our cohort. This discrepancy, however, could relate to differences in testing strategies, where we sequenced all nonrepetitive coding sequences of $N E B$, whereas only a single frequent deletion was assessed in the previous study. ${ }^{1}$ Also, the genetic background may influence the distribution of genetic etiology.

We identified the genetic etiology in $56 \%$ of patients. Two recent studies (same group) describing retrospective data in mostly pediatric patients reported the genetic cause in $67 \%-79 \% .^{1,2}$ Our lower diagnostic rate can partly be explained by methods of selecting patients, as we included many patients with unspecific myopathic biopsies and that subgroup only had a genetic etiology identified in $21 \%$ of cases. Leaving the patients with unspecific myopathic histology out, we found a genetic etiology in 63\% if CFTD/T1 was included, and $83 \%$ without CFTD/T1. Differences in the success rate of identifying genetic etiology are therefore most likely caused by differences in histology among the included cohorts. An alternative explanation might be that our patients were older than those in previous studies and that those who die before age 5 years often have mutations in ACTA1 or $M T M 1,{ }^{2}$ which are relatively easy to identify. Finally, the genetic background may vary as discussed above.

The chance of identifying genetic etiology was highly dependent on histology. A much higher percentage with a "specific" histology like cores, NM, 


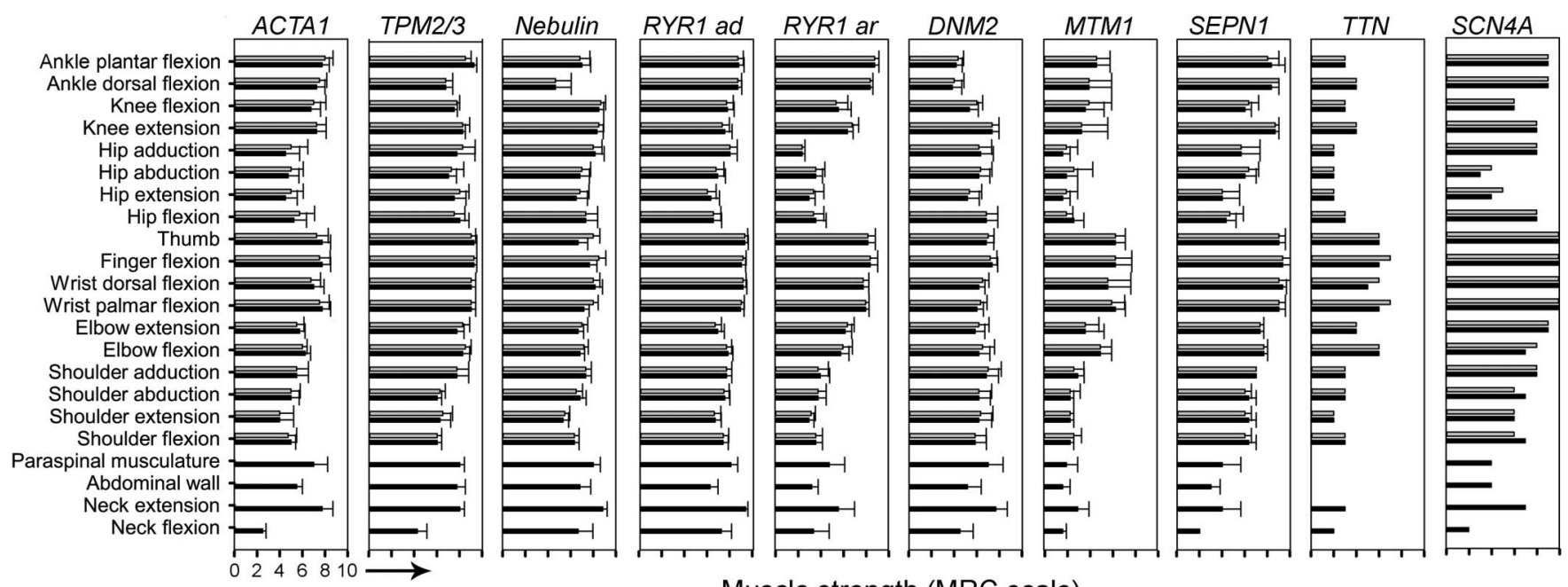

Muscle strength (MRC scale)

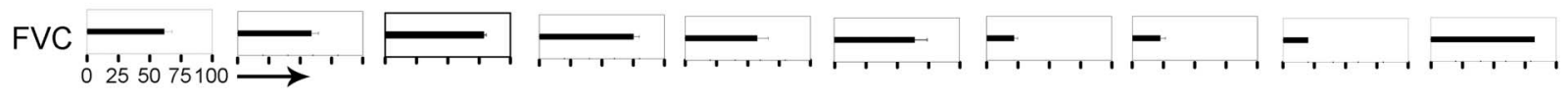

Percentage of expected normal values

For Medical Research Council (MRC); gray bars left side, black bars right side. Bottom: forced vital capacity (FVC) in percentage of expected normal values.

or CNM had a genetic etiology identified. In the subgroup with confirmed CM and NM, genetic etiology was identified in $100 \%$. However, the specificity of $\mathrm{CM}$ is not $100 \%$, as 1 patient with desmin mutation also had NM. NM in patients with desmin mutations has only been reported in a few cases, ${ }^{16}$ but was attributed to the known protein accumulation in desminopathy. "Specific histology" for CM, however, was only found in 3/10 patients with a phenotype compatible with CM, but with alternative genetic diagnoses (CMS, desminopathy, ColVI, or XII). A patient with selenoprotein deficiency had nemaline bodies in 2 muscle fibers. Although this might be an incidental finding, this patient was grouped together with the NMs.

The detailed physical examination performed in this study suggests some new clinical clues to the genetic etiology. In the genetically resolved nemaline/CFTD/T1 group, ACTA1 patients were generally weaker than $N E B$ patients. ${ }^{17}$ However, in contrast to the $N E B$ patients, patients affected by TPM2 and 3 aberrations had a disproportionately higher respiratory affection, and half of the $N E B$ patients had almost paralytic ankle dorsiflexion coexisting with MRC 7-8 in proximal muscles and a relatively preserved hand function. The preferential weakness of ankle dorsiflexors in some $N E B$ patients is well established. ${ }^{18,19}$ The only patient with combined CFTD and pronounced ophthalmoplegia had recessive $R Y R 1$ disease. The recessive $R Y R 1$ patients also exhibited a marked proximal-distal gradient in weakness, which was shared by the MTM1 patients, but the MTM1 patients had a much more noticeable respiratory affection. Severe ophthalmoplegia was, in agreement with a recent consensus statement, ${ }^{19}$ exclusively observed with recessive $R Y R 1, D N M 2$, and MTM1 mutations. CCD was almost synonymous with dominant RYR1 mutations, and these patients had much the same extremity affection as SEPN1 patients, and both groups had a tendency to scoliosis. The SEPN1 patients, however, had much more respiratory and axial involvement as known for this condition. Mutations in DNM2 lead to CNM with ptosis and a lower extremity distal affection in all cases, but otherwise a relatively mild phenotype. Although these clinical clues are not $100 \%$ consistent, we believe that they contribute importantly when planning a genetic test strategy or interpreting test results.

Ten patients were initially judged to have a phenotype compatible with $\mathrm{CM}$, but turned out to have mutation in a gene not strictly belonging to the genes recognized as genes inducing CM; 3 had mutations in the collagen VI gene, 3 in the collagen XII gene, 2 in the desmin gene, and 2 had CMS. The delineation of CM is not generally agreed upon, but we have, in line with others, chosen not to include collagen myopathies, as they typically have a progressive course and more contractures than other CMs. On follow-up, the widespread contractures in the 3 collagen VI patients led to the identification of mutations in the 
collagen VI genes, and in the patients with desmin mutations, the course showed to be much more progressive than was the impression at the initial visit. Hence, in retrospect, the collagen VI myopathies and the desmin patients should not have been included. By contrast, there were no red flags in the phenotype of the collagen XII patients and the patients with CMSs. The prevalence would not have been significantly different if these patients were included, as the total patient number compared to the complete population is still very small.

In contrast to most previous studies, we included many adult patients with very early-onset weakness. We confirmed that for the majority, the disease course is nonprogressive and many patients are still engaged in an active work life.

Taken together, this study adds new knowledge about the geographic variation in prevalence, distribution of subtypes, and clinical characteristics in an older population with CM that may influence strategies for diagnostic testing and counseling of patients.

\section{AUTHOR CONTRIBUTIONS}

Nanna Witting: study concept and design, acquisition of data, analysis and interpretation of data, and drafting of manuscript. Ulla Werlauff: study concept and design, acquisition of data, analysis and interpretation of data, and critical revision of manuscript for intellectual content. Morten Duno: acquisition of data, analysis and interpretation of data, and critical revision of manuscript for intellectual content. John Vissing: study concept and design, analysis and interpretation of data, and critical revision of manuscript for intellectual content.

\section{STUDY FUNDING}

This study was sponsored by the Novo Nordisk Foundation.

\section{DISCLOSURE}

Dr. Witting received research support from the Danish Council for Independent Research in Medical Sciences, the Augustinus Foundation, the Jascha Foundation, the Novo Nordisk Foundation, and the Gangsted Foundation. Ms. Werlauff and Mr. Duno report no disclosures. Dr. Vissing has received research support, travel support, and speaker honoraria from Genzyme/Sanofi and Ultragenyx Pharmaceuticals; has served on the editorial boards of Neuromuscular Disorders and the Journal of Neuromuscular Diseases; has received research support from aTyr Pharma, the Danish Medical Research Council, the Augustinus Foundation, the Novo Nordisk Foundation, and the Lundbeck Foundation; and has acted as a consultant on advisory boards for Genzyme/Sanofi, Lundbeck, Ultragenyx Pharmaceuticals, Novo Nordisk, aTyr Pharma, Sarepta, and Alexion Pharmaceuticals within the last 3 years. Go to Neurology.org/ng for full disclosure forms.

Received September 16, 2016. Accepted in final form January 30, 2017.

\section{REFERENCES}

1. Maggi L, Scoto M, Cirak S, et al. Congenital myopathies -clinical features and frequency of individual subtypes diagnosed over a 5-year period in the United Kingdom. Neuromuscul Disord 2013;23:195-205.

2. Colombo I, Scoto M, Manzur AY, et al. Congenital myopathies: natural history of a large pediatric cohort. Neurology 2015;84:28-35.
3. Pelin K, Hilpela P, Donner K, et al. Mutations in the nebulin gene associated with autosomal recessive nemaline myopathy. Proc Natl Acad Sci USA 1999;96:23052310.

4. Brandt A, Schleithoff L, Jurkat-Rott K, Klingler W, Baur C, Lehmann-Horn F. Screening of the ryanodine receptor gene in 105 malignant hyperthermia families: novel mutations and concordance with the in vitro contracture test. Hum Mol Genet 1999;8:2055-2062.

5. Carmignac V, Salih MA, Quijano-Roy S, et al. C-terminal titin deletions cause a novel early-onset myopathy with fatal cardiomyopathy. Ann Neurol 2007;61:340-351.

6. Hackman P, Vihola A, Haravuori H, et al. Tibial muscular dystrophy is a titinopathy caused by mutations in TTN, the gene encoding the giant skeletal-muscle protein titin. Am J Hum Genet 2002;71:492-500.

7. Romero NB, Clarke NF. Congenital myopathies. Handb Clin Neurol 2013;113:1321-1336.

8. Brooke MH, Griggs RC, Mendell JR, Fenichel GM, Shumate JB, Pellegrino RJ. Clinical trial in Duchenne dystrophy: I: the design of the protocol. Muscle Nerve 1981;4: 186-197.

9. Lohmueller KE, Sparso T, Li Q, et al. Whole-exome sequencing of 2,000 Danish individuals and the role of rare coding variants in type 2 diabetes. Am J Hum Genet 2013;93:1072-1086.

10. Klein A, Lillis S, Munteanu I, et al. Clinical and genetic findings in a large cohort of patients with ryanodine receptor 1 gene-associated myopathies. Hum Mutat 2012;33: 981-988.

11. Hughes MI, Hicks EM, Nevin NC, Patterson VH. The prevalence of inherited neuromuscular disease in Northern Ireland. Neuromuscul Disord 1996;6:69-73.

12. Darin N, Tulinius M. Neuromuscular disorders in childhood: a descriptive epidemiological study from western Sweden. Neuromuscul Disord 2000;10:1-9.

13. Amburgey K, McNamara N, Bennett LR, McCormick ME, Acsadi G, Dowling JJ. Prevalence of congenital myopathies in a representative pediatric United States population. Ann Neurol 2011;70:662-665.

14. Sveen ML, Schwartz M, Vissing J. High prevalence and phenotype-genotype correlations of limb girdle muscular dystrophy type 2I in Denmark. Ann Neurol 2006;59: 808-815.

15. Norwood FL, Harling C, Chinnery PF, Eagle M, Bushby $\mathrm{K}$, Straub V. Prevalence of genetic muscle disease in Northern England: in-depth analysis of a muscle clinic population. Brain 2009;132:3175-3186.

16. Hong D, Wang Z, Zhang W, et al. A series of Chinese patients with desminopathy associated with six novel and one reported mutations in the desmin gene. Neuropathol Appl Neurobiol 2011;37:257-270.

17. North KN, Laing NG. Skeletal muscle alpha-actin diseases. Adv Exp Med Biol 2008;642:15-27.

18. Wallgren-Pettersson C, Lehtokari VL, Kalimo H, et al. Distal myopathy caused by homozygous missense mutations in the nebulin gene. Brain 2007;130:1465-1476.

19. North KN, Wang CH, Clarke N, et al. Approach to the diagnosis of congenital myopathies. Neuromuscul Disord 2014;24:97-116

20. Bohm J, Biancalana V, Dechene ET, et al. Mutation spectrum in the large GTPase dynamin 2, and genotypephenotype correlation in autosomal dominant centronuclear myopathy. Hum Mutat 2012;33:949-959. 
21. Citirak G, Witting N, Duno M, Werlauff U, Petri H, Vissing J. Frequency and phenotype of patients carrying TPM2 and TPM3 gene mutations in a cohort of $94 \mathrm{pa}-$ tients with congenital myopathy. Neuromuscul Disord 2014;24:325-330.

22. Werlauff U, Petri H, Witting N, Vissing J. Frequency and phenotype of myotubular myopathy amongst Danish patients with congenital myopathy older than 5 years. J Neuromusc Dis 2015;2:167-174.
23. Witting N, Werlauff U, Duno M, Vissing J. Prevalence and phenotypes of congenital myopathy due to alpha-actin 1 gene mutations. Muscle Nerve 2016;53:388-393.

24. Zaharieva IT, Thor MG, Oates EC, et al. Loss-of-function mutations in SCN4A cause severe foetal hypokinesia or "classical" congenital myopathy. Brain 2016;139:674-691.

25. Kaplan JC, Hamroun D. The 2015 version of the gene table of monogenic neuromuscular disorders (nuclear genome). Neuromuscul Disord 2014;24:1123-1153. 


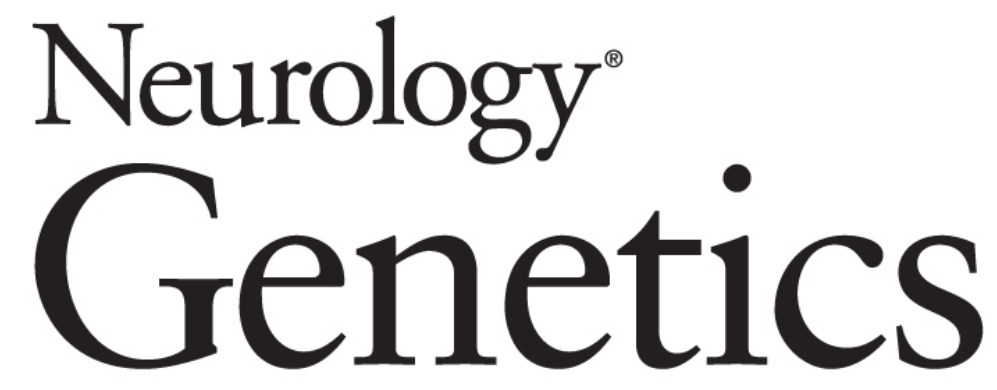

Phenotypes, genotypes, and prevalence of congenital myopathies older than 5 years in Denmark

Nanna Witting, Ulla Werlauff, Morten Duno, et al. Neurol Genet 2017;3;

DOI 10.1212/NXG.0000000000000140

This information is current as of March 21, 2017

Neurol Genet is an official journal of the American Academy of Neurology. Published since April 2015, it is an open-access, online-only, continuous publication journal. Copyright Copyright @ 2017 The Author(s).

Published by Wolters Kluwer Health, Inc. on behalf of the American Academy of Neurology. All rights reserved. Online ISSN: 2376-7839.

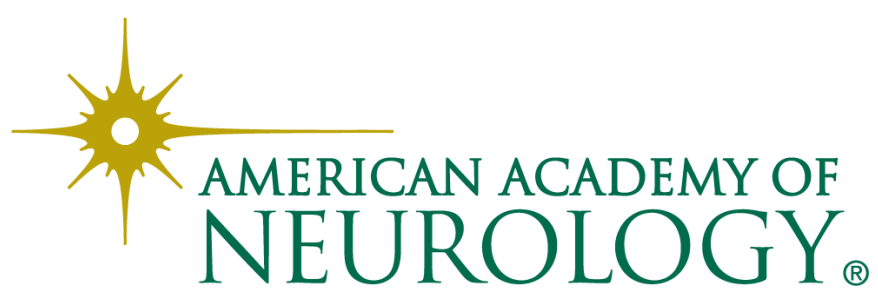




\section{Updated Information \& Services}

References

Citations

Subspecialty Collections

Permissions \& Licensing

Reprints including high resolution figures, can be found at: http://ng.neurology.org/content/3/2/e140.full.html

This article cites 25 articles, 1 of which you can access for free at: http://ng.neurology.org/content/3/2/e140.full.html\#\#ref-list-1

This article has been cited by 2 HighWire-hosted articles: http://ng.neurology.org/content/3/2/e140.full.html\#\#otherarticles

This article, along with others on similar topics, appears in the following collection(s):

All Clinical Neurology

http://ng.neurology.org//cgi/collection/all_clinical_neurology All Genetics

http://ng.neurology.org//cgi/collection/all_genetics

Muscle disease

http://ng.neurology.org//cgi/collection/muscle_disease

Information about reproducing this article in parts (figures,tables) or in its entirety can be found online at:

http://ng.neurology.org/misc/about.xhtml\#permissions

Information about ordering reprints can be found online:

http://ng.neurology.org/misc/addir.xhtml\#reprintsus

Neurol Genet is an official journal of the American Academy of Neurology. Published since April 2015, it is an open-access, online-only, continuous publication journal. Copyright Copyright ( 2017 The Author(s). Published by Wolters Kluwer Health, Inc. on behalf of the American Academy of Neurology. All rights reserved. Online ISSN: 2376-7839.

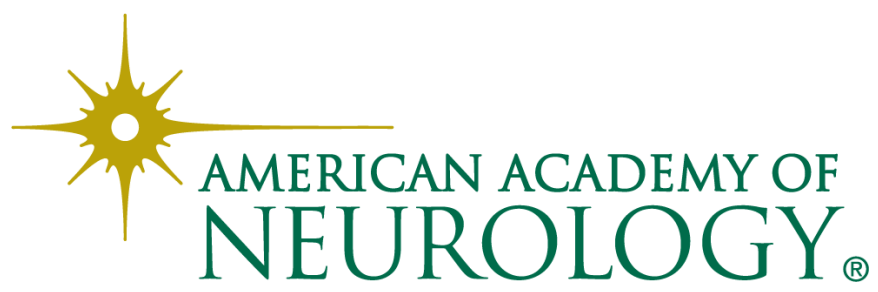

